\title{
Kinematic Synthesis of Planar 4-Bar Path Generators for Finite Line Positions
}

\author{
Gökhan Kiper ${ }^{1[0000-0001-8793-724 X]}$ and Eres Söylemez ${ }^{\text {[0000-0003-2324-959X] }}$ \\ ${ }^{1}$ İzmir Institute of Technology, Urla, İzmir 35430, Turkey \\ ${ }^{2}$ Middle East Technical University, Ankara 06800, Turkey \\ gokhankipereiyte.edu.tr
}

\begin{abstract}
Although the kinematic synthesis of planar function, point-path and motion generators are vastly studied in the literature, surprisingly synthesis of line-path generators is not formulized in detail. This study presents the formulization of the planar 4-bar line-path generator synthesis problem for up to 5 homologous positions. Numerical examples for 3 and 4 line positions are presented for the illustration of the formulations.
\end{abstract}

Keywords: Kinematic Synthesis, 4-Bar Mechanism, Line-Path Generation.

\section{Introduction}

Kinematic synthesis of planar linkages are classically studied under three main categories: motion generation, function generation, and point-path generation and path generation with prescribed timing [1-2]. There are some studies on kinematic synthesis of spatial line-path generator linkages [3-8]. Homologous lines passing through a point or tangent to a circle in plane are issued in [5]. However according to the authors' knowledge, there is no study on synthesis of planar line-path generator 4-bar linkages.

In some applications such as grinding, filing and packaging it is necessary to design a linkage, end-effector of which needs to be enveloping a given planar curve or a surface with no torsion. In other words, a line is sought on the end-effector which remains tangent to a given planar curve. If the envelope of lines can be approximately represented by finitely many lines, the problem can be cast into the design of a planar linkage, end-effector of which passes through finitely many lines. That is, a line on the coupler link instantaneously coincides with some lines attached to the fixed link at different instants. This paper presents the derivations of the necessary formulation for the kinematic synthesis of planar 4-bar linkages for specified three and four line positions. It is shown that the line generation problem can be mathematically converted into a motion generation problem and solved similarly. Numerical examples are presented to illustrate the formulations. 


\section{Problem Definition and Formulation}

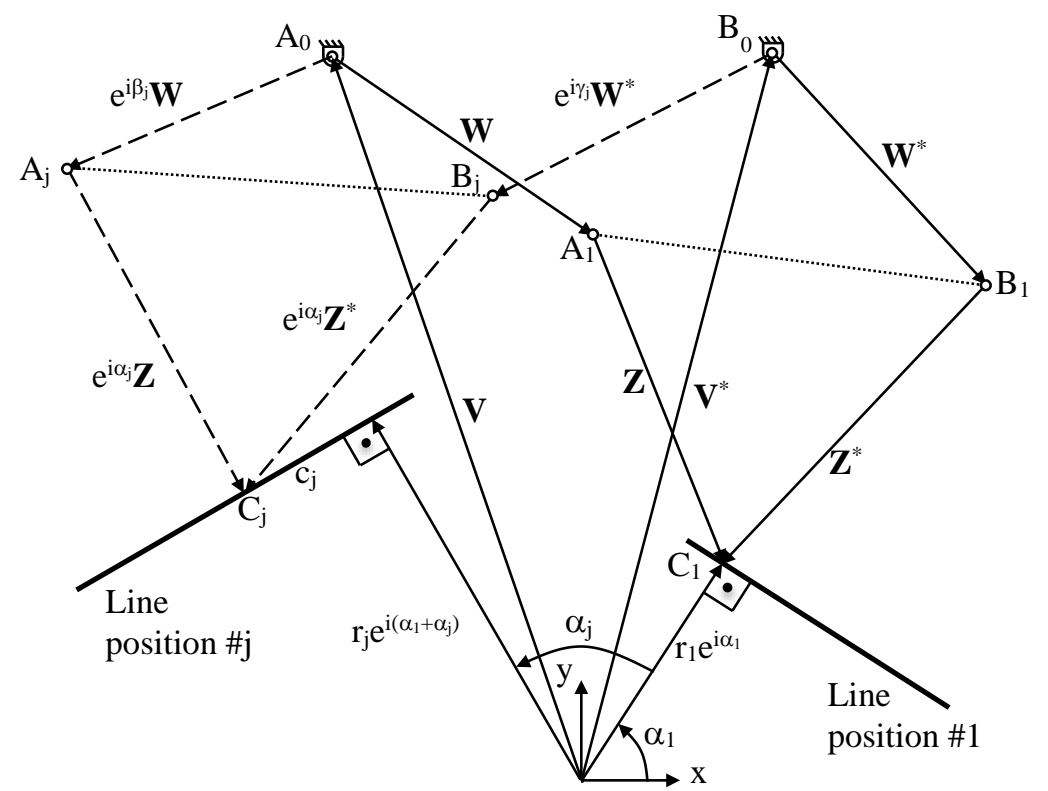

Fig. 1. Kinematic diagram for the synthesis

The homologous positions $\# 1, \# 2, \ldots, \# \mathrm{n}$ of a line are represented by their distance to the origin $r_{1}, r_{2}, \ldots, r_{n}$ and angles $\alpha_{1}, \alpha_{2}, \ldots, \alpha_{n}$ with respect to a selected reference frame as shown in Fig. 1. When a 4-bar linkage $\mathrm{A}_{0} \mathrm{ABB}_{0}$ is used for the task, dyads $\mathrm{A}_{0} \mathrm{AC}$ and $\mathrm{B}_{0} \mathrm{BC}$ can be designed separately. $\mathrm{ABC}$ represents the coupler link of the linkage. Point $\mathrm{C}$ is chosen to be a coupler point which is instantaneously coincident with the foot of the perpendicular drawn from the origin to the line at position \#1. For dyad $\mathrm{A}_{0} \mathrm{AC}$ at position \#1 of the line:

$$
\mathbf{V}+\mathbf{W}+\mathbf{Z}=\mathrm{r}_{1} \mathrm{e}^{\mathrm{i} \alpha_{1}}
$$

For simplifying the solution, let the coordinate frame be set such that the first line is on the y-axis, i.e. $r_{1}=0$ and $\alpha_{1}=0$. Let link $A_{0} A$ be rotated by an angle $\beta_{j}$ from position $\# 1$ to position $\# \mathrm{j}$. Then for $\mathrm{j}=2, \ldots, n$

$$
\left.\begin{array}{c}
\mathbf{V}+\mathbf{W}+\mathbf{Z}=\mathbf{0} \\
\mathbf{V}+\mathrm{e}^{i \beta_{j}} \mathbf{W}+\mathrm{e}^{i \alpha_{j}} \mathbf{Z}=\left(r_{j}+i c_{j}\right) e^{i \alpha_{j}}
\end{array}\right\}\left(e^{i \beta_{j}}-1\right) \mathbf{W}+\left(e^{i \alpha_{j}}-1\right) \mathbf{Z}=\left(r_{j}+i c_{j}\right) e^{i \alpha_{j}}
$$

$r_{j}$ and $\alpha_{j}$ are given. If $\beta_{j}$ values are assumed, $\mathbf{W}, \mathbf{Z}$ and $c_{j}$ 's can be solved linearly. However, note that $\mathrm{c}_{\mathrm{j}}$ 's will appear in diad $\mathrm{B}_{0} \mathrm{BC}$ equations as well. Let rotations of link $\mathrm{B}_{0} \mathrm{~B}$ be $\gamma_{\mathrm{j}}$ from position $\# 1$ to position $\# \mathrm{j}$. Then 


$$
\left.\begin{array}{c}
\mathbf{V}^{*}+\mathbf{W}^{*}+\mathbf{Z}^{*}=0 \\
\mathbf{V}^{*}+\mathbf{W}^{*} \mathrm{e}^{\mathrm{i} \gamma_{j}}+\mathbf{Z}^{*} \mathrm{e}^{\mathrm{i} \alpha_{j}}=\left(\mathrm{r}_{\mathrm{j}}+\mathrm{ic} \mathrm{c}_{\mathrm{j}}\right) \mathrm{e}^{\mathrm{i} \alpha_{\mathrm{j}}}
\end{array}\right\}\left(\mathrm{e}^{\mathrm{i} \gamma_{j}}-1\right) \mathbf{W}^{*}+\left(\mathrm{e}^{\mathrm{i} \alpha_{j}}-1\right) \mathbf{Z}^{*}=\left(\mathrm{r}_{\mathrm{j}}+\mathrm{ic} \mathrm{c}_{\mathrm{j}}\right) \mathrm{e}^{\mathrm{i} \alpha_{\mathrm{j}}}
$$

If $\gamma_{j}$ values are assumed, $\mathbf{W}^{*}, \mathbf{Z}^{*}$ and $c_{j}$ 's can be solved linearly. Keeping all the unknowns, the number of scalar equations versus the number of unknowns for different number of finite positions are listed in Table 1.

Table 1. Number of scalar equations v.s. the number of unknowns

\begin{tabular}{|c|c|c|c|}
\hline $\mathrm{n}$ & $\begin{array}{c}\text { Number of } \\
\text { equations }\end{array}$ & Unknowns & $\begin{array}{l}\text { Number of free } \\
\text { parameters: }\end{array}$ \\
\hline 2 & 4 & $\mathrm{~W}_{\mathrm{x}}, \mathrm{W}_{\mathrm{y}}, \mathrm{Z}_{\mathrm{x}}, \mathrm{Z}_{\mathrm{y}}, \mathrm{c}_{2}, \beta_{2}, \mathrm{~W}_{\mathrm{x}}^{*}, \mathrm{~W}_{\mathrm{y}}^{*}, \mathrm{Z}_{\mathrm{x}}^{*}, \mathrm{Z}_{\mathrm{y}}^{*}, \gamma_{2}$ & 7 \\
\hline 3 & 8 & $\mathrm{~W}_{\mathrm{x}}, \mathrm{W}_{\mathrm{y}}, \mathrm{Z}_{\mathrm{x}}, \mathrm{Z}_{\mathrm{y}}, \mathrm{c}_{2}, \mathrm{c}_{3}, \beta_{2}, \beta_{3}, \mathrm{~W}_{\mathrm{x}}^{*}, \mathrm{~W}_{\mathrm{y}}^{*}, \mathrm{Z}_{\mathrm{x}}^{*}, \mathrm{Z}_{\mathrm{y}}^{*}, \gamma_{2}, \gamma_{3}$ & 6 \\
\hline 4 & 12 & $\begin{array}{c}\mathrm{W}_{\mathrm{x}}, \mathrm{W}_{\mathrm{y}}, \mathrm{Z}_{\mathrm{x}}, \mathrm{Z}_{\mathrm{y}}, \mathrm{c}_{2}, \mathrm{c}_{3}, \mathrm{c}_{4}, \beta_{2}, \beta_{3}, \beta_{4} \\
\mathrm{~W}_{\mathrm{x}}^{*}, \mathrm{~W}_{\mathrm{y}}^{*}, \mathrm{Z}_{\mathrm{x}}^{*}, \mathrm{Z}_{\mathrm{y}}^{*}, \gamma_{2}, \gamma_{3}, \gamma_{4}\end{array}$ & 5 \\
\hline 5 & 16 & $\begin{array}{c}W_{x}, W_{y}, Z_{x}, Z_{y}, c_{2}, c_{3}, c_{4}, c_{5}, \beta_{2}, \beta_{3}, \beta_{4}, \beta_{5} \\
W_{x}^{*}, W_{y}^{*}, Z_{x}^{*}, Z_{y}^{*}, \gamma_{2}, \gamma_{3}, \gamma_{4}, \gamma_{5} \\
\end{array}$ & 4 \\
\hline 6 & 20 & $\begin{array}{c}W_{x}, W_{y}, Z_{x}, Z_{y}, c_{2}, c_{3}, c_{4}, c_{5}, c_{6}, \beta_{2}, \beta_{3}, \beta_{4}, \beta_{5}, \beta_{6} \\
W_{x}^{*}, W_{y}^{*}, Z_{x}^{*}, Z_{y}^{*}, \gamma_{2}, \gamma_{3}, \gamma_{4}, \gamma_{5}, \gamma_{6}\end{array}$ & 3 \\
\hline 7 & 24 & $\begin{array}{c}W_{x}, W_{y}, Z_{x}, Z_{y}, c_{2}, c_{3}, c_{4}, c_{5}, c_{6}, c_{7}, \beta_{2}, \beta_{3}, \beta_{4}, \beta_{5}, \beta_{6}, \beta_{7}, \\
W_{x}^{*}, W_{y}^{*}, Z_{x}^{*}, Z_{y}^{*}, \gamma_{2}, \gamma_{3}, \gamma_{4}, \gamma_{5}, \gamma_{6}, \gamma_{7}\end{array}$ & 2 \\
\hline 8 & 28 & $\begin{array}{c}\mathrm{W}_{\mathrm{x}}, \mathrm{W}_{\mathrm{y}}, \mathrm{Z}_{\mathrm{x}}, \mathrm{Z}_{\mathrm{y}}, \mathrm{c}_{2}, \mathrm{c}_{3}, \mathrm{c}_{4}, \mathrm{c}_{5}, \mathrm{c}_{6}, \mathrm{c}_{7}, \mathrm{c}_{8}, \beta_{2}, \beta_{3}, \beta_{4}, \beta_{5}, \beta_{6}, \beta_{7}, \\
\beta_{8}, \mathrm{~W}_{\mathrm{x}}^{*}, \mathrm{~W}_{\mathrm{y}}^{*}, \mathrm{Z}_{\mathrm{x}}^{*}, \mathrm{Z}_{\mathrm{y}}^{*}, \gamma_{2}, \gamma_{3}, \gamma_{4}, \gamma_{5}, \gamma_{6}, \gamma_{7}, \gamma_{8}\end{array}$ & 1 \\
\hline 9 & 32 & $\begin{array}{c}\mathrm{W}_{\mathrm{x}}, \mathrm{W}_{\mathrm{y}}, \mathrm{Z}_{\mathrm{x}}, \mathrm{Z}_{\mathrm{y}}, \mathrm{c}_{2}, \mathrm{c}_{3}, \mathrm{c}_{4}, \mathrm{c}_{5}, \mathrm{c}_{6}, \mathrm{c}_{7}, \mathrm{c}_{8}, \mathrm{c}_{9}, \beta_{2}, \beta_{3}, \beta_{4}, \beta_{5}, \beta_{6}, \\
\beta_{7}, \beta_{8}, \beta_{9}, \mathrm{~W}_{\mathrm{x}}^{*}, \mathrm{~W}_{\mathrm{y}}^{*}, \mathrm{Z}_{\mathrm{x}}^{*}, \mathrm{Z}_{\mathrm{y}}^{*}, \gamma_{2}, \gamma_{3}, \gamma_{4}, \gamma_{5}, \gamma_{6}, \gamma_{7}, \gamma_{8}, \gamma_{9}\end{array}$ & 0 \\
\hline
\end{tabular}

If $\mathrm{c}_{\mathrm{j}}$ 's are freely selected, the problem becomes equivalent to motion generation problem. For 2 line positions, In addition to $c_{2}, \beta_{2}, \gamma_{2}$, any two of $W_{x}, W_{y}, Z_{x}, Z_{y}$ for dyad $\mathrm{A}_{0} \mathrm{AC}$ and any two of $\mathrm{W}^{*}{ }_{\mathrm{x}}, \mathrm{W}^{*}{ }_{\mathrm{y}}, \mathrm{Z}_{\mathrm{x}}{ }_{\mathrm{x}}, \mathrm{Z}_{\mathrm{y}}^{*}$ for dyad $\mathrm{B}_{0} \mathrm{BC}$ can be freely chosen. Ground pivot specification (specification of $-\mathbf{W}-\mathbf{Z}$ and $-\mathbf{W}^{*}-\mathbf{Z}^{*}$ ) is also possible. For 3 line positions once $c_{2}, c_{3}, \beta_{2}, \beta_{3}, \gamma_{2}, \gamma_{3}$ are assumed, $\mathbf{W}, \mathbf{Z}, \mathbf{W}^{*}$ and $\mathbf{Z}^{*}$ can be solved linearly. The 2 - and 3 -line position problems can also be solved graphically.

For 4-line positions if $\mathrm{c}_{2}, \mathrm{c}_{3}, \mathrm{c}_{4}, \beta_{2}$, and $\gamma_{2}$ are assumed, solution of $\mathbf{W}, \mathbf{Z}, \mathbf{W}^{*}, \mathbf{Z}^{*}$, $\beta_{3}, \beta_{4}, \gamma_{3}, \gamma_{4}$ is the same as the 4-position motion generation problem. For dyad $\mathrm{A}_{0} \mathrm{AC}$

$$
\begin{aligned}
& \left(e^{i \beta_{2}}-1\right) \mathbf{W}+\left(e^{i \alpha_{2}}-1\right) \mathbf{Z}=\left(r_{2}+i c_{2}\right) e^{i \alpha_{2}} \\
& \left(e^{i \beta_{3}}-1\right) \mathbf{W}+\left(e^{i \alpha_{3}}-1\right) \mathbf{Z}=\left(r_{3}+i c_{3}\right) e^{i \alpha_{3}} \Rightarrow\left[\begin{array}{cc}
e^{i \beta_{2}}-1 & e^{i \alpha_{2}}-1 \\
e^{i \beta_{3}}-1 & e^{i \alpha_{3}}-1 \\
\left(e^{i \beta_{4}}-1\right) \mathbf{W}+\left(e^{i \alpha_{4}}-1\right) \mathbf{Z}=\left(r_{4}+i c_{4}\right) e^{i \alpha_{4}}
\end{array}\right]\left[\begin{array}{c}
\mathbf{W} \\
\mathbf{Z}
\end{array}\right]=\left[\begin{array}{c}
\left(r_{2}+i c_{2}\right) e^{i \alpha_{2}} \\
\left(r_{3}+i c_{3}\right) e^{i \alpha_{3}} \\
\left(r_{4}+i c_{4}\right) e^{i \alpha_{4}}
\end{array}\right]
\end{aligned}
$$

These equations have a solution provided that the augmented matrix is singular:

$$
\left|\begin{array}{ccc}
e^{i \beta_{2}}-1 & e^{i \alpha_{2}}-1 & \left(r_{2}+i c_{2}\right) e^{i \alpha_{2}} \\
e^{i \beta_{3}}-1 & e^{i \alpha_{3}}-1 & \left(r_{3}+i c_{3}\right) e^{i \alpha_{3}} \\
e^{i \beta_{4}}-1 & e^{i \alpha_{4}}-1 & \left(r_{4}+i c_{4}\right) e^{i \alpha_{4}}
\end{array}\right|=\Delta_{34}\left(r_{2}+i c_{2}\right)-\Delta_{24}\left(r_{3}+i c_{3}\right)+\Delta_{23}\left(r_{4}+i c_{4}\right)=0
$$


where

$$
\begin{aligned}
& \Delta_{34}=\mathrm{e}^{\mathrm{i} \alpha_{2}}\left[\left(\mathrm{e}^{\mathrm{i} \beta_{3}}-1\right)\left(\mathrm{e}^{\mathrm{i} \alpha_{4}}-1\right)-\left(\mathrm{e}^{\mathrm{i} \beta_{4}}-1\right)\left(\mathrm{e}^{\mathrm{i} \alpha_{3}}-1\right)\right] \\
& \Delta_{24}=\mathrm{e}^{\mathrm{i} \alpha_{3}}\left[\left(\mathrm{e}^{\mathrm{i} \beta_{2}}-1\right)\left(\mathrm{e}^{\mathrm{i} \alpha_{4}}-1\right)-\left(\mathrm{e}^{\mathrm{i} \beta_{4}}-1\right)\left(\mathrm{e}^{\mathrm{i} \alpha_{2}}-1\right)\right] \\
& \Delta_{23}=\mathrm{e}^{\mathrm{i} \alpha_{4}}\left[\left(\mathrm{e}^{\mathrm{i} \beta_{2}}-1\right)\left(\mathrm{e}^{\mathrm{i} \alpha_{3}}-1\right)-\left(\mathrm{e}^{\mathrm{i} \beta_{3}}-1\right)\left(\mathrm{e}^{\mathrm{i} \alpha_{3}}-1\right)\right]
\end{aligned}
$$

The solution to this problem is well known. However, with a different set of freely chosen parameters, the problem is varied from the classical motion generation problem. Let $\beta_{2}, \beta_{3}, \beta_{4}, \gamma_{2}$ and either one of $c_{2}, c_{3}$ and $c_{4}$ be specified. Then, the remaining two can be linearly solved from Eq. (5). Then, $\mathbf{W}$ and $\mathbf{Z}$ are solved linearly from any of the three complex equations in Eq. (4). Once $c_{2}, c_{3}$ and $c_{4}$ are either specified or solved from the first dyad, for the second dyad, the solution of $\mathbf{W}^{*}, \mathbf{Z}^{*}, \gamma_{3}$ and $\gamma_{4}$ for given $\gamma_{2}$ is the same as solving the 4-position motion generation problem. There will be two sets of solutions.

For 5 line positions we can assume $\mathrm{c}_{2}, \mathrm{c}_{3}, \mathrm{c}_{4}, \mathrm{c}_{5}$ and solve for $\mathrm{W}_{\mathrm{x}}, \mathrm{W}_{\mathrm{y}}, \mathrm{Z}_{\mathrm{x}}, \mathrm{Z}_{\mathrm{y}}, \beta_{2}, \beta_{3}$, $\beta_{4}, \beta_{5}, W^{*}{ }_{x}, W^{*}{ }_{y}, Z_{x}^{*}, Z^{*}, \gamma_{2}, \gamma_{3}, \gamma_{4}$ and $\gamma_{5}$ in the same way of solving the 5 -position motion generation problem. An alternative solution is to assume $\beta_{2}, \beta_{3}, \beta_{4}, \beta_{5}$ in the first dyad and linearly solve for $c_{2}, c_{3}, c_{4}, c_{5}$ from the following complex singular determinant equations:

$$
\left|\begin{array}{ccc}
\mathrm{e}^{\mathrm{i} \beta_{2}}-1 & \mathrm{e}^{\mathrm{i} \alpha_{2}}-1 & \left(\mathrm{r}_{2}+\mathrm{ic} \mathrm{c}_{2}\right) \mathrm{e}^{\mathrm{i} \alpha_{2}} \\
\mathrm{e}^{\mathrm{i} \beta_{3}}-1 & \mathrm{e}^{\mathrm{i} \alpha_{3}}-1 & \left(\mathrm{r}_{3}+\mathrm{ic}_{3}\right) \mathrm{e}^{\mathrm{i} \alpha_{3}} \\
\mathrm{e}^{\mathrm{i} \beta_{4}}-1 & \mathrm{e}^{\mathrm{i} \alpha_{4}}-1 & \left(\mathrm{r}_{4}+\mathrm{ic}_{4}\right) \mathrm{e}^{\mathrm{i} \alpha_{4}}
\end{array}\right|=0 \text { and }\left|\begin{array}{ccc}
\mathrm{e}^{\mathrm{i} \beta_{2}}-1 & \mathrm{e}^{\mathrm{i} \alpha_{2}}-1 & \left(\mathrm{r}_{2}+\mathrm{ic} \mathrm{c}_{2}\right) \mathrm{e}^{\mathrm{i} \alpha_{2}} \\
\mathrm{e}^{\mathrm{i} \beta_{3}}-1 & \mathrm{e}^{\mathrm{i} \alpha_{3}}-1 & \left(\mathrm{r}_{3}+\mathrm{ic} \mathrm{c}_{3}\right) \mathrm{e}^{\mathrm{i} \alpha_{3}} \\
\mathrm{e}^{\mathrm{i} \beta_{5}}-1 & \mathrm{e}^{\mathrm{i} \alpha_{5}}-1 & \left(\mathrm{r}_{4}+\mathrm{ic} \mathrm{c}_{4}\right) \mathrm{e}^{\mathrm{i} \alpha_{5}}
\end{array}\right|=0
$$

These singular determinants yield two complex equations which are linear in $c_{2}, c_{3}, c_{4}$, $c_{5}$. Once $c_{2}, c_{3}, c_{4}, c_{5}$ are solved, the second dyad can be determined as in the 5position motion generation problem.

For $\mathrm{n}>5$ line positions, there are $9-\mathrm{n}$ free parameters to choose, $2(n+1)$ unknowns for each dyad, where $n-1$ many $c_{j}$ 's are common for both dyads. Since there are 2(n-1) equations for each dyad, once 9-n $<4$ parameters are specified, the remaining parameters cannot be solved for a dyad without taking the other dyad into considerations. That is, the dyad equations become coupled and are highly nonlinear. An analytical solution is unlikely.

\section{$3 \quad$ Examples}

As an example, three and four positions of a line enveloping a parabola is worked out. Consider the parabola $y=x^{2}+x / 2$ and three tangent lines at points $(0,0),(1,1.5)$ and $(3,10.5)$ (all units are $\mathrm{mm})$. The corresponding distance $r_{j}$ and angle $\alpha_{j}$ values for $\mathrm{j}=2,3$ are listed in Table 2. An Excel simulation environment is created. After several trials $c_{2}, c_{3}, \beta_{2}, \beta_{3}, \gamma_{2}, \gamma_{3}$ values are chosen as listed in Table 2. Using Eqs. (2) and (3), $\mathbf{W}, \mathbf{Z}, \mathbf{W}^{*}, \mathbf{Z}^{*}$ vector coordinates and link lengths $\mathrm{a}_{1}=\left|\mathbf{W}+\mathbf{Z}-\mathbf{W}^{*}-\mathbf{Z}^{*}\right|$, 
$\mathrm{a}_{2}=|\mathbf{W}|, \mathrm{a}_{3}=\left|\mathbf{Z}-\mathbf{Z}^{*}\right|, \mathrm{a}_{4}=\left|\mathbf{W}^{*}\right|, \mathrm{b}_{3}=|\mathbf{Z}|$ and $\mathrm{c}_{3}=\left|\mathbf{Z}^{*}\right|$ are determined and listed in Table 2. The resulting 4 - bar linkage and the family of coupler lines are depicted in Fig. 2.

Table 2. Parameters for the 3-line positions example

\begin{tabular}{c|c|c|c|c|c|c|c|c|c}
$\mathrm{r}_{2}$ & $\mathrm{r}_{3}$ & $\alpha_{2}$ & $\alpha_{3}$ & $\mathrm{c}_{2}$ & $\mathrm{c}_{3}$ & $\beta_{2}$ & $\beta_{3}$ & $\gamma_{2}$ & $\gamma_{3}$ \\
\hline 0.371 & 1.369 & $41.64^{\circ}$ & $54.69^{\circ}$ & 0.6 & 1 & $70^{\circ}$ & $140^{\circ}$ & $30^{\circ}$ & $60^{\circ}$ \\
\hline \hline $\mathbf{W}$ & $\mathbf{Z}$ & $\mathbf{W}^{*}$ & $\mathbf{Z}^{*}$ & $\mathrm{a}_{1}$ & $\mathrm{a}_{2}$ & $\mathrm{a}_{3}$ & $\mathrm{a}_{4}$ & $\mathrm{~b}_{3}$ & $\mathrm{c}_{3}$ \\
\hline $\begin{array}{c}-1.405 \\
-\mathrm{i} 1.969\end{array}$ & $\begin{array}{c}1.431 \\
+\mathrm{i} 0.367\end{array}$ & $\begin{array}{c}-0.878 \\
-\mathrm{i} 0.321\end{array}$ & $\begin{array}{c}1.768 \\
-\mathrm{i} 1.112\end{array}$ & 1.186 & 0.935 & 1.516 & 2.419 & 2.088 & 1.477
\end{tabular}
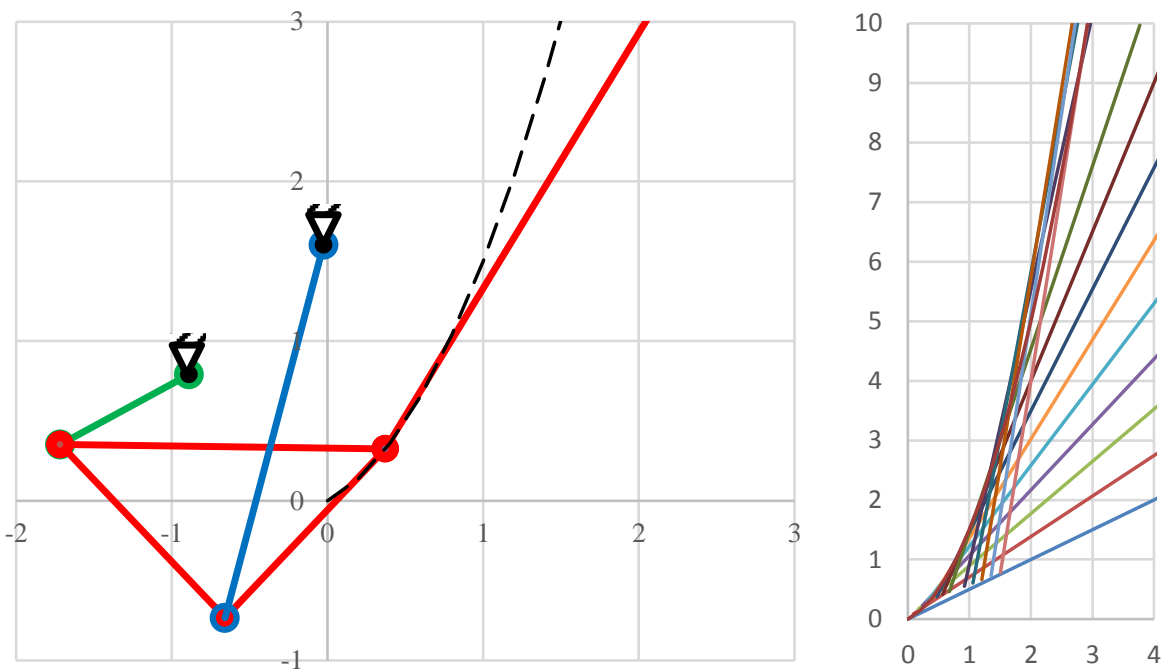

Fig. 2. The designed 4-bar linkage for 3-line positions and the family of coupler lines

Next, let's consider the same parabola and four tangent lines at points $(0,0),(1,1.5)$, $(3,10.5)$ and $(4,18)$. In addition to the $r_{j}$ and $\alpha_{j}$ values in Table $2, r_{4}$ and $\alpha_{4}$ are given in Table 3. This time, a Mathcad simulation environment is created. After several trials $c_{2}, \beta_{2}, \beta_{3}, \beta_{4}, \gamma_{2}$ values are chosen as listed in Table 2. Using Eqs. (4) and (5), $c_{3}$, $\mathrm{c}_{4}, \gamma_{3}, \gamma_{4}, \mathbf{W}, \mathbf{Z}, \mathbf{W}^{*}, \mathbf{Z}^{*}$ vector coordinates and link lengths $\mathrm{a}_{1}=\left|\mathbf{W}+\mathbf{Z}-\mathbf{W}^{*}-\mathbf{Z}^{*}\right|$, $\mathrm{a}_{2}=|\mathbf{W}|, \mathrm{a}_{3}=\left|\mathbf{Z}-\mathbf{Z}^{*}\right|, \mathrm{a}_{4}=\left|\mathbf{W}^{*}\right|, \mathrm{b}_{3}=|\mathbf{Z}|, \mathrm{c}_{3}=\left|\mathbf{Z}^{*}\right|$ are determined and listed in Table 3. The resulting 4-bar linkage is presented in Fig. 3.

Table 3. Speciflied, chosen and computed parameters for the 4-line positions example

\begin{tabular}{c|c|c|c|c|c|c|c|c|c|c}
$\mathrm{r}_{4}$ & $\alpha_{4}$ & $\beta_{2}$ & $\beta_{3}$ & $\beta_{4}$ & $\mathrm{c}_{2}$ & $\gamma_{2}$ & $\mathrm{c}_{3}$ & $\mathrm{c}_{4}$ & $\gamma_{3}$ & $\gamma_{4}$ \\
\hline 0.371 & $54.69^{\circ}$ & $30^{\circ}$ & $70^{\circ}$ & $100^{\circ}$ & 0.8 & $90^{\circ}$ & 0.444 & 0.279 & $142.03^{\circ}$ & $176.22^{\circ}$ \\
\hline \hline $\mathbf{W}$ & $\mathbf{Z}$ & $\mathbf{W}^{*}$ & $\mathbf{Z}^{*}$ & $\mathrm{a}_{1}$ & $\mathrm{a}_{2}$ & $\mathrm{a}_{3}$ & $\mathrm{a}_{4}$ & $\mathrm{~b}_{3}$ & $\mathrm{c}_{3}$ \\
\hline $\begin{array}{c}-0.910 \\
-\mathrm{i} 0.500\end{array}$ & $\begin{array}{c}1.096 \\
-\mathrm{i} 1.583\end{array}$ & $\begin{array}{c}0.331 \\
+\mathrm{i} 0.790\end{array}$ & $\begin{array}{c}0.518 \\
-\mathrm{i} 2.852\end{array}$ & 1.190 & 1.043 & 1.394 & 0.858 & 1.926 & 2.899
\end{tabular}




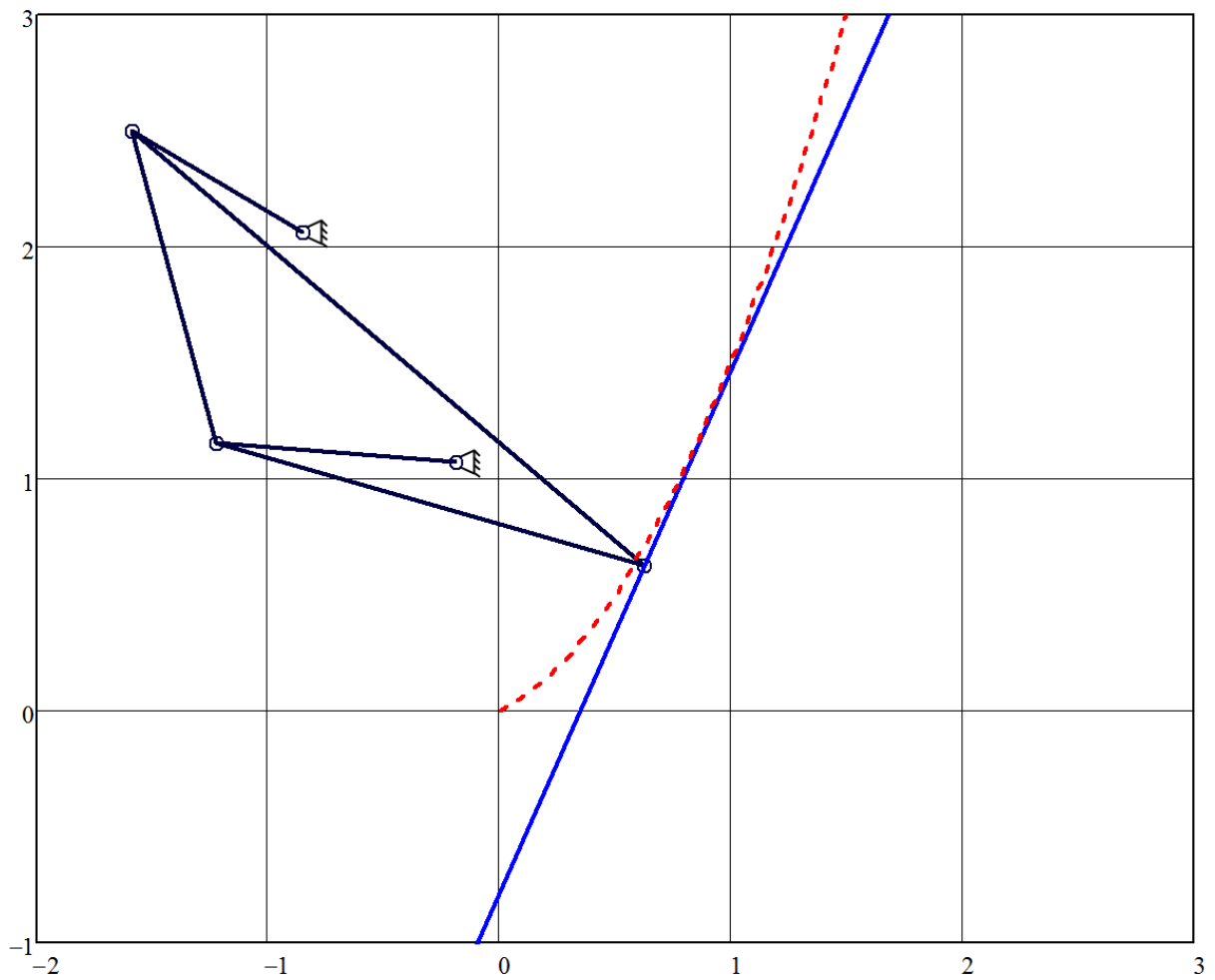

Fig. 3. The designed 4-bar linkage for 4-line positions

\section{Conclusions}

In this study the planar 4-bar line-path generator synthesis problem is formulized for up to 5 homologous positions. As in function and point-path generation problems, the line-path generation problem also can be mathematically cast into motion generation problem. Numerical examples for 3 and 4 line positions are provided.

\section{Acknowledgments}

The authors thank to Prof. Bernard Roth for his comments and suggestions.

\section{References}

1. Sandor, G. N., Erdman, A. G.: Advanced Mechanism Design: Analysis and Synthesis. Vol. 2. Prentice Hall, Englewood Cliffs, NJ (1984). 
2. McCarthy, J. M., Soh, G. S.: Geometric Design of Linkages. 2nd edn. Springer, New York (2010).

3. Chen, P., Roth, B.: A unified theory for the finitely and infinitesimally separated position problems of kinematic synthesis. Journal of Engineering for Industry 91(1), 203-208 (1969).

4. Tsai, L-W., Roth, B.: Incompletely specified displacements: geometry and spatial linkage synthesis, Transaction of ASME, Journal of Engineering for Industry 95(B), 603-611 (1973).

5. Bottema, O., Roth, B.: Theoretical Kinematics. North Holland, Amsterdam (1979).

6. Parkin, I. A.: A third conformation with the screw systems: finite twist displacements of a directed line and point. Mechanism and Machine Theory 27(2), pp. 177-188 (1992).

7. Huang, C.: On the finite screw system of the third order associated with a revoluterevolute chain, Journal of Mechanical Design 116, pp. 875-883 (1994).

8. Huang, C., Huang, B.: Spatial generalization of the planar path generation problem. In: Kecskeméthy, A., Müller, A. (eds.) Computational Kinematics. pp. 117-13. Springer, Berlin (2009). 